\title{
The Journal of Comparative Neurology and Psychology
}

\begin{tabular}{lll}
\hline volume XIV Number 5 \\
\hline
\end{tabular}

\section{RETROGRADE DEGENERATION IN THE CORPUS}

CALLOSUM OF THE WHITE RAT.

By S. Walter Ranson.

( From the Neurological Laboratory of the Universily of Chicago and the Anatomical Laboratory of St. Louis Unizersity.)

With Plate VII.

SUMMARY OF THE LITERATURE.

It was maintained by Waller (6) and those who immediately followed him that the end of the nerve fiber attached to the cell body did not degenerate as the result of section of the fiber. Evidence has, however, been steadily accumulating to show that this view is incorrect. The facts bearing on this point have been brought together by FLeming ( 1 ), KLIPPEL and DURante (2), and van Gehuchten (4). These authors review the literature in great detail ; but only the briefest summary will be given here, and this will be based chiefly on the excellent review by van Gehuchten.

The first observations not in harmony with the law of WaLler were made upon cases of long standing amputation. Atrophy of the ventral and dorsal root fibers and of the part of the spinal cord associated with the nerves of the amputated limb has been found in these cases. (Dickinson, '68, Vulpian, '68, Hayem and Gilbert, '84, Marinesco, '92, and others). Experimental amputations of the limbs of animals, involving section of the peripheral nerves, have confirmed these observations (Vulpian, '69 and Hayem, '73), and shown the presence of fibers with fragmented myelin in the central ends of the cut nerves. Degenerating fibers were also found in the ventral and dorsal nerve roots and in the dorsal fasciculi of the cord. (DARKsChewitsch, '92 and Moschaew, '93). 
382 Journal of Comparative Neurology and Psychology.

The second group of observations opposed to the statement formulated by WALLER has been derived from experiments intended to locate the nuclei of origin of the motor nerves. The tearing out of motor nerves is followed by atrophy or disappearance of their intramedullary roots and nuclei of origin. (Literature summarized by Forel, '87.) A true degeneration of the central ends of these fibers can be demonstrated by the method of Marchi (Bregmann, '92, Darkschewitsch, '92).

In the central nervous system a retrograde or cellulipetal degeneration has been observed in seven localities. A descending degeneration has been seen in the optic radiation after excision of its terminal ramifications in the occipital cortex (VON Monakow, '84, Moeli, '93) and in the medial lemniscus after injury to the central gyri (von Monakow, '85, Greiwe, '94, Klippel and Durante, '95). The retrograde degeneration in these tracts differs in some cases histologically from WaLLERIAN degeneration, the axis cylinder remaining intact while the myelin sheath disappears, although in most cases it resembles true secondary degeneration very closely. Often this cellulipetal degeneration is very extensive, involving the majority of the fibers of the tract. For these reasons, the downward degeneration in the optic radiation and in the lemniscus cannot be adduced as evidence for a double pathway. Similar observations have been made on the pyramidal tract, which in certain cases has degenerated cephalad after a transverse lesion of the spinal cord (Willamson, '93, Raymond, '94).

VAN Genuchten has shown that degeneration of the proximal part of injured nerve fibers may occur in the middle cercbellar peduncle, in the fibers passing from the nucleus ruber to the lateral fasciculus of the spinal cord, in the fibers passing from the nucleus of DeITtrs to the anterior fasciculus of the cord, and in those from the cells of the formatio reticularis of the pons and medulla to the antero-lateral fasciculus of the cord. That retrograde degeneration occurs in these tracts and not merely the WaLLERIAN degeneration of a second pathway, is supported by the facts that the changes in question do not ap- 
pear until fifteen days after WALLERIAN degeneration has begun and that the cells of origin of these tracts disappear.

OBSERVATIONS ON THE WHITE RAT.

Introduction and Summary.

In a previous paper the writer (3) called attention to the complete degeneration of the splenium of the corpus callosum in a young rat after deep incision of the occipital lobe, and reference was made to a similar observation by voN GUDDEN (5). If this degeneration had occurred according to WALLER's law, about half the normal number of fibers should have been present on each side of the lesion. But in this case all the fibers had disappeared. This means that the fibers underwent serious alterations in both directions from the point of injury. This observation taken by itself only shows the absence of the myelin sheath in the proximal portion of the injured fiber, as no stains were used which would demonstrate the presence or absence of naked axis cylinders. Since there were no medullated fibers in the corpus callosum at the time of the operation the absence of myelin might be interpreted as due to an arrest of development. But further observations made on older rats have shown that a true degeneration closely resembling the WALLERIAN type may occur in the proximal portions of severed fibers.

The chief difference between the changes in the proximal and the distal portions is that the latter pass more rapidly through the stages of fragmentation, solution and absorption, these changes affecting the whole extent of the severed portion at the same time; while in the proximal portions the changes occur somewhat later and may involve only the part of the fiber nearest the point of injury. Thus forty-five days after a wound is made in the medullated corpus callosum of young rats ( 2 to 70 days of age) the ordinary WALLERIAN degeneration has run its full course and the resulting debris is entirely absorbed. But at this time fragmentation of myelin may be distinctly seen in the proximal portions of the severed fibers, affecting especially the part in the vicinity of the lesion. 
384 Journal of Comparative Neurology and Psychology.

\section{Operative Technique.}

Through the occipital portion of the corpus callosum in rats of various ages $(0.5,3,7,2 \mathrm{I}, 30,40,60,70$ days old), an incision was made in the left cerebral hemisphere about one millimeter to the left of the great longitudinal sinus and two millimeters frontal to the lateral sinus, in such a way that a wound one and a half millimeters long and two or three millimeters deep was made in the posterior part of the occipital lobe, parallel to the midplane. The animals were killed forty-five days after the operation. In each case serial frontal sections through the left occipital lobe were prepared according to the PAL-WeIGert technique.

\section{Results.}

In so simple an operation asepsis is not difficult to obtain. The wounds were covered with collodion and remained well protected until healing had taken place. The animals recovered rapidly and after twenty-four hours appeared to be perfectly normal. During the entire subsequent period they were in excellent physical condition, and equaled in weight the rats of the same age in the laboratory. In only one case did the postmortem examination show any trace of inflammatory reaction. In this rat slight adhesions were present between the dura and the brain scar, but these produced no appreciable effect on the results.

The mildest form of the cellulipetal degeneration is found in the brain of the oldest rat (seventy days old at the time of operation). Except for the comparatively narrow band of scar tissue, there is no area in which complete degeneration of all the fibers has taken place. Portions of many fibers must have been cut off from their cells of origin, and these portions have no doubt undergone complete WALLERIAN degeneration, although the debris resulting from their disintegration has been entirely absorbed. Thus on each side of the cicatrix there is brain tissue which at first sight appears normal. But on closer examination many of the fibers in the immediate vicinity of the scar are found to differ from the normal in that they have an 
irregular contour, being very much constricted at certain points and swollen into beads at others. Most of these fibers stain well by the PaL-Weigert method. Their myelin has not been gathered into droplets nor been to any appreciable extent absorbed. These beaded fibers are not to be found more than two millimeters on either side of the line of incision since the cellulipetal degeneration had extended only a short distance along the fiber.

In a rat ten days younger (sixty days old at the time of operation) there are considerable areas of degeneration in the substantia alba. Figure I, " $a$ " shows a Y-shaped area of faint staining extending medialward from the scar. There is also a slender unstained band extending lateralward. In these areas the cellulipetal degeneration has progressed somewhat farther than in the case previously described. Near the line of incision all the myelin has been absorbed, leaving only the faintest outline of the fibers visible. Figure 2 is a drawing of a small portion of the degenerated area represented at " $a$ " in Figure $\mathbf{I}$. In addition to the beaded fibers described in the previous case there are many that are faintly stained, some of which contain minute droplets of myelin. There are also many large faintly stained drops of myelin which do not appear to be connected with fibers.

In the rat which was thirty days old at the time of the operation, the process of cellulipetal degeneration has increased in intensity and extended farther along the fibers. Figure 3, " $a$ ", shows a comparatively wide band in the substantia alba faintly stained because of the disappearance of most of the fibers. Near the line of incision this disappearance of fibers is complete; only a few scattered drops of myelin are visible. Farther lateralward (Fig. 4) are fibers in the process of disintegration, some swollen and beaded, other faintly and irregularly stained. Still farther lateralward these give place to normally stained, smoothly contoured fibers.

Changes similar to those described above were found in the brain of a rat operated on when twenty-one days old; but here fibers were found degenerating at a much greater dis- 


\section{Journal of Comparative Neurology and Psychology.}

tance from the point of injury. Fig. 5 shows that most of these fibers are in the last stages of degeneration. They are very irregular in shape, and their outlines are only faintly visible. They stain faintly except in a few places, where there still remain minute globules of myelin. They are broken up into short segments, so that no fiber can be followed for any considerable distance. A few droplets of free myelin are still unabsorbed.

In order to interpret these results it is necessary to bear in mind the condition of medullation in the corpus callosum of the young rat. WATson (7) has shown that medullation in this region begins about the fourteenth day. Thus, fibers cut in the operation performed on the twenty-first day are both structurally and functionally very immature; and the rapidity with which they degenerate is, no doubt, closely related to this condition. By the seventieth day the neurones have attained a greater de. gree of stability, which shows itself in the much more limited retrograde degeneration. When the operation was made on or before the seventh day of age no medullated fibers were cut. because there were none present. This would account for the fact that in examining the preparations of these brains no fibers with disintegrating myelin sheaths were found. There are, however, areas which are almost devoid of fibers, although they exhibit a few that are slender, normally contoured and well stained. On the basis of the earlier experiments (3) these are to be explained as having developed since the injury. Complete degeneration is best seen in the corpus callosum of the rat op. erated on when twelve hours old, which has been figured in the Journal of Comparative Neurology, Vol. XIII, Plate VII, Figures 4 and 7 . Much the same condition is seen in the corpus callosum of the next older rat ( 3 days); but in this and the one operated on at the seventh day the wound is situated so far posteriorly, that the picture is complicated by the presence of fibers curving backward into the occipital lobe. These fibers, coming from in front and passing backward and outward, cross the zone which would otherwise be free from fibers.

As has been already stated, this paucity of medullated fibers in the corpus callosum, so evident in the youngest rat, 
might be interpreted as due to a failure of development of the medullary sheaths about the central portions of the severed axones; or it might be due to the complete disappearance of these axones. But when one takes into consideration the retrograde degeneration seen in these fibers in the older rats, there can be little doubt that the degeneration is of the same nature in these younger animals and that the entire neurone has undergone disintegration.

If it is remembered that all these animals were allowed to live for one month and a half after the operation, it will be seen from what has been said that the intensity of the cellulipetal degeneration exhibited in any portion of the section depends upon two variables, the age of the animal and the distance between the point of observation and the point of injury. In the oldest rat the process was confined to a small portion of the fiber near the lesion. As we pass down the series from the oldest to the youngest animal, the degencration increases in intensity in the vicinity of the lesion, and extends to a greater distance from the point of injury. The complete degeneration of the fibers in the older rats takes place only in the immediate vicinity of the lesion, but in the youngest animal the fibers degenerate completely throughout their entire length.

There are several respects in which the changes here described differ from the WaLlekian degeneration. In typical secondary degeneration the myelin begins to liquify at the sixth day and is largely absorbed before the twentieth. But in the milder cases of the degeneration here described there are many fibers remaining after forty-five days which differ from the normal only in the presence of a few beadlike swellings, the rest of the fiber remaining normal in contour and staining properties. And in these milder cases there are no fibers present in which the myelin has undergone liquefaction. It is evident that the secondary degeneration, which must have occurred in the portions of the fibers separated from their cell bodies, has run its full course and the resulting debris been entirely absorbed. Otherwise we could not account for the complete absence in some of these cases of fibers in the last stages of degeneration. 


\section{Journal of Comparative Neurology and Psychology.}

The degeneration which is seen beginning in certain fibers 45 days after the lesion is, therefore, a process commencing after the ordinary secondary degeneration is complete. Another feature which distinguishes retrograde degeneration from secondary degeneration is the fact that the latter occurs simultaneously throughout the entire length of the severed part, while the former may involve only a small part of a fiber in the immediate vicinity of the lesion.

A word may be said regarding the nature and cause of this degeneration of the proximal part of injured fibers. VAN Genuchten (4) has shown that associated with these changes in the fiber leading to its disintegration, there is a chromatolysis of the cells of origin resulting, in certain cases, in their complete destruction. In those neurones in which this occurs he believes that the degeneration of the fiber follows on the death of the cell and proceeds down the fiber toward its terminals. For this reason, he objects to the use of the term "retrograde" in designating this type of degeneration, because it indicates that the degeneration begins at the point of injury and proceeds toward the cell body.

It is, however, quite conceivable that the evidence of this disintegration of the neurone should be found first in the portion of the neurone farthest separated from the nucleus, namely at the tip of the fiber near the lesion. As a matter of fact many of the preparations upon which this paper is based show a degeneration in the end near the lesion while the rest of the fiber appears normal. In some cases beginning near the lesion and tracing the fiber toward the cell of origin one may see all the stages of degeneration; at first the fiber stains faintly because of the absorption of its myelin, then it is found to be well stained but of irregular contour and finally it takes on a perfectly normal appearance. It seems clear, therefore, that in this case at least the process is a true retrograde or cellulipetal degeneration. 


\section{Bibllography.}

1. Fleming, R. A. '97. Edinburg Med. Jour., Vol. XLIII, pp. 49-279.

2. Klippel et Durante. '95. Revue de Médecine, Vol. XV, pp. I, 142, 343, 574,655 .

3. Ranson, S. W. 'o3. Jour. Comp. Neurol., Vol. XIII, p. 186.

4. Van Gehuchten, A. 'O3. Le Névraxe, Vol. V, p. 3.

5. Von Gudden, B. ' 89 . Gesammelte und hinterlassene Abhandlungen, p. 130. Königl. Universitäts-Druckerei in Würzburg.

6. Waller. ' 52 . Comp . Rend. de I' Academie des Sciences, Vol. XXXIV, p. 582.

7. Watson, John B. 'o3. Animal Education: an experimental study on the psychical development of the white rat, correlated with the growth of its nervous system. The University of Chicago Press, Chicago.

\section{DESCRIPTION OF PLATE VII.}

The drawings, made by Mr. A. B. STREedAin, represent retrograde degeneration in the corpus callosum of white rats, killed forty-five days after the making of an incision in the occipital lobe. They were made from PAL-WEIGERT preparations of frontal sections through the occipital lobe.

Figure $t$ is from a rat operated on when sixty days old. $a$ and $b$, degenerating areas.

$c$, line of incision.

$m$, medial surface of the hemisphere.

Figure $a$ is from the same preparation as the former drawing. It represents the appearance under the oil immersion lens of a small area designated by "a," Figure I.

Figure 3 is from a rat operated on when thirty days old. The lettering is the same as in Figure 1.

Figure 4 is from the same preparation as Figure 3. It represents a small area at " $a$ " seen.under the oil immersion lens.

Figure 5 was drawn with the aid of the oil immersion from the degenerating corpus callosum of a rat operated on when 21 days old. 